\title{
Virtual Human Model Representation and Driven Deformation Methods
}

\author{
Hongwei $\mathrm{An}^{1}$, Renjie $\mathrm{Xu}^{1, *}$ and Luohua Zhao ${ }^{1}$ \\ ${ }^{1}$ Academy of Armored Forces Engineering ,Beijing 10072, China \\ ${ }^{*}$ Corresponding author
}

Keywords: Virtual Human, Driven Deformation, LOD.

\begin{abstract}
In this paper, we will summarize the existing three-dimensional virtual human body model representation and drive deformation method with real-time and realistic requirements as a starting point, and propose a combination of surface model and skeleton driving to realize virtual human motion drawing.
\end{abstract}

\section{Introduction}

The model representation and driving deformation of 3D virtual human body is the basis of the research of large-scale virtual population motion rendering technology, which directly affects the efficiency of large-scale virtual population movement drawing. The 3D virtual human model geometric representation is directly related to skin deformation, and different virtual human model structures have different skin deformation methods. At present, the representation of three-dimensional virtual human body model mainly has rod-like, surface, entity, multi-level model [1].

\section{Virtual human model representation method}

\subsection{Rod body model}

The rod-shaped model is the earliest virtual body geometry model. As shown in Fig. 1.1, the rod-shaped model represents the human body as a simple linker of segments and joints. The kinematic model is used to simulate the image. This simple skeleton model only can simulate the general movement of the human body, does not involve the skin of the representation and deformation.

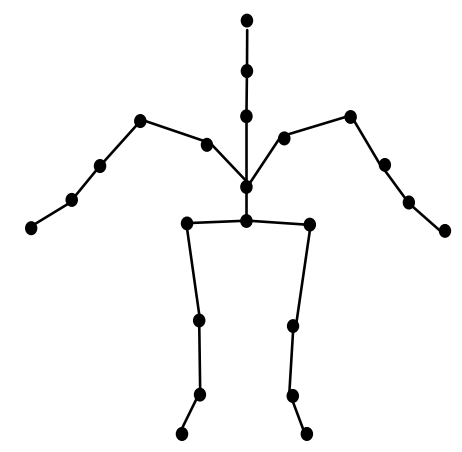

Figure 1. 1Figure headings

\subsection{Surface model}

The surface model consists of two levels, which is a hierarchical structure model. These two levels are the skeleton layer and the skin layer, as shown in Fig.1.2. The skeletal layer is sorted by the hierarchical relationship of the skeletons of the human body and forms the basis of the three-dimensional virtual human movement. The skin layer is composed of a flat sheet to simulate the geometric shape of the skin. The skin is attached to the skeleton and its deformation is driven by the underlying skeleton. The advantage of the surface model is fast, the disadvantage is that without 
considering the human anatomy, it is difficult to achieve realistic simulation results. Improve the surface model of skin deformation is one of the current research focus.

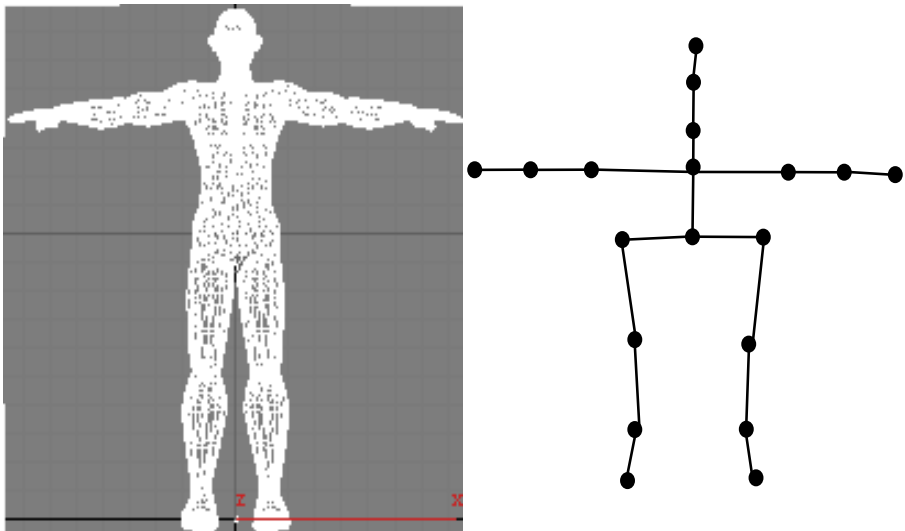

Figure 1.2 Surface model

\subsection{Entity model}

The solid model uses a simple set of entities to simulate the structure and shape of the body, such as spheres, cylinders, ellipsoids, etc., using the implicit surface display method, the use of light projection method to determine the body surface skin, the calculation of large, complex modeling process. Solid model has not been widely applied to the transition to a multi-level model. Fig.1.3 is an example of a solid model.

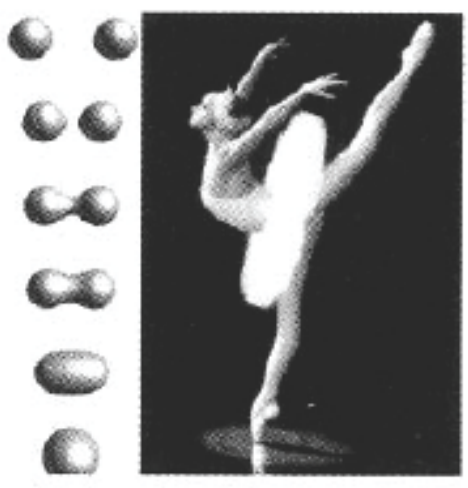

Figure 1.3 Solid model

\subsection{Multi-level model}

Multi-level model usually uses the skeleton layer to support the middle layer and skin layer, the closest to the human anatomy, the middle layer contains bone, muscle, adipose tissue. The skin is not simply attached to the skeleton, the dynamic squeezing and stretching effect from the underlying skeletal movement and adipose tissue movement, muscle swelling. Multi-level modeling method can construct a very realistic three-dimensional virtual human body model. But the model complexity is high, the body deformation when the calculation is large.

\section{Virtual human skin deformation drive method and model animation technology}

Based on the surface model, the skeleton - driven skin deformation method and the skeleton skin animation technique are proposed. The combination of the surface model and the skeleton driving deformation is proposed to realize the virtual human motion drawing, and the improvement will be made on this basis. 


\subsection{Virtual human skin deformation drive method}

Based on the surface model, the virtual human motion-driven algorithm has a lot of rigidity due to the extensive application of the surface model. There are rigid deformation, local operator deformation, deformation based on case interpolation and skeleton-driven deformation [1]. Rigid deformation of the various parts of the body as a rigid body, through the skeleton of the various joints of the chain control relationship to achieve a variety of human animation, the method is fast, the model of each vertex to accept a matrix transformation to get the final position, But at the joint prone to fold and other distortion of the phenomenon. The method of deformation based on local operator is a method of skin deformation which is applied to non-rigid body early. By using the continuous deformation function of joint value, the deformation state of the vertex near each joint is realized, and the method can produce realistic effect, But each joint uses a specific deformation function, can not be further editing and control. The method of deformation based on case interpolation uses hardware equipment, such as scanners, to obtain real human body shape data, or use existing realistic model resources to obtain a set of examples of standards that are fused between instances to achieve realistic posture Skin morphology, without too much consideration of human anatomy, the disadvantage is the great workload and the interpolation function is not clear.

Skeleton-driven deformation method [2] (Skeleton Driven Deformation, SDD; also known as skin, Skinning Mesh; or vertex blending, Vertex Blending; or skeleton subspace deformation, Skeleton-Subspace Deformation, etc.)[3][4] is the real-time realistic deformation method First, has received hardware support. The main idea is that the control of the deformation of the skeleton is completed by each joint, and each vertex gets the position of the joint through the matrix transformation (usually the rotation matrix) at each joint, and the final position is obtained in a weighted form. which is:

$$
\boldsymbol{P}_{v}=\sum \boldsymbol{a}_{i} \square \boldsymbol{P}_{i}=\sum \boldsymbol{a}_{i} \square \boldsymbol{M}_{i} \square \boldsymbol{M}_{\text {offset }-i} \square \boldsymbol{P}_{o}
$$

Where the original vertex is multiplied by the matrix to the local space of the joint $i$, and the result of the influence of the joint $\mathrm{i}$ is obtained by multiplying the transformation matrix of the local space, and the final position is weighted by the weight a $(\Sigma=1)$. The results obtained using this method are shown in Figure 1.4. The use of SDD as a deformation is easy to form a special case of distortion, for example, due to the typical rotation of different joints and different to the time, will lead to joints at the skin twist phenomenon, and some other cases of skin elongation or contraction More serious distortion. However, due to its high computational efficiency, there has been a very large improvement over rigid deformation, and the appropriate treatment can greatly avoid the above phenomenon, and therefore is particularly suitable for large-scale virtual population movement display One of the driving methods. In this paper, based on the surface model representation method, the method of skeleton driving deformation is proposed, and the method of surface model and skeleton driving deformation is proposed to realize virtual human motion drawing.

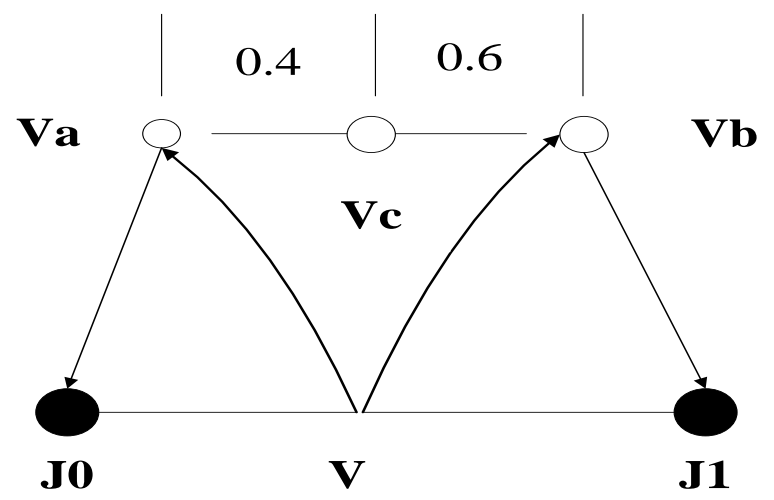

Figure 1.4 Skeleton-driven deformation (SSD) algorithm 


\subsection{Real-time character animation technology}

Character animation refers to human animation. In the traditional three-dimensional virtual human animation, the producers need to carefully analyze the role of the action, the role of the movement by the people manually, frame by frame to adjust the key frame data, very cumbersome, the production process often mistakes, The animation is not satisfactory. In computer animation, it is quite difficult to establish interesting and realistic virtual human movements and to control them, usually taking a compromise between the effectiveness and complexity of the control. For a fixed joint structure, commonly used optimization method to automatically generate a dynamic control system.

At present, real-time virtual human body animation technology has three main categories, namely, joint animation, single grid animation and bone skin animation.

\subsubsection{Joint animation}

In the joint animation, the role is composed of a number of independent parts, each part corresponds to a separate grid model. For example, a human body model can be composed of the head, upper body, upper left arm, left hand, right upper arm, right hand, left thigh, left foot, right thigh, right foot and other parts. One of the parts may be another part of the child node, but also another part of the parent node. As in the above human model, the upper right arm is the upper node of the child, the upper body is the body of the child nodes. The desired animation can be achieved by changing the relative position between the different parts (such as displacement, angle, etc.). The advantages of this type of animation are as follows: First, in the animation sequence key frame only need to store the relative changes between the nodes, the animation file occupies a small space; Second, can achieve many complex animation effects, if the application supports reverse power Learning can also dynamically implement new animation effects outside the pre-stored animation sequence. This kind of animation also has many shortcomings: First, the role model is a hierarchical model, must be traversed from the root node of all the ancestral nodes of the node to calculate the model world transformation to obtain a part of the position relative to the world coordinates; Is the combination of different parts often have a very obvious seams, which will seriously affect the reality of the model. As shown in Figure 2.1:

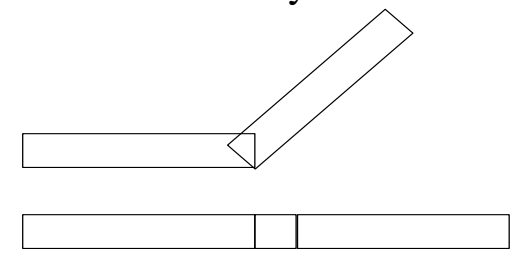

(a)

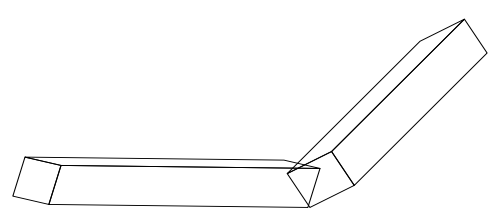

(b)

Figure 2.1 Break between joints

\subsubsection{A single grid animation}

In a single grid animation, the character consists of a complete grid model. The new position of each vertex that makes up the grid is recorded in the key frame of the animation sequence. The position of each vertex in the grid model is directly changed by interpolating between adjacent key frames to achieve the animation effect. Relative to the joint animation, the role of a single grid model animation looks more real, there will not be joint animation problems faced by the joints, and get the model grid vertices in the world coordinates of the calculation of the location is also very small. However, the adaptability of this type of animation is very weak, the role is difficult to real-time calculation to interact with the environment in order to obtain pre-stored animation sequence outside the animation effect. Second, because the keyframe needs to store all the vertex information in the grid model, the animation file occupies a particularly large space.

\subsubsection{Bone Skin Animation}


In the skeletal skin animation, a character consists of a single mesh model as a skin and a skeleton organized at a certain level. Bone skin animation can be seen as a combination of joint animation and single grid model animation. Bone hierarchy describes the structure of the character, such as the different parts of the joint animation, skeletal skin animation in the skeleton in accordance with the characteristics of the role of a hierarchical structure. Adjacent bones are connected by joints, for the relative movement, by changing the displacement between the adjacent bones, angle, so that the role of the skeleton to make different actions to achieve different animation effects. The skin is covered as a mesh on the bones, reflecting the appearance of the character. The skin is not a fixed rigid mesh, but a deformable mesh that can be changed under the influence of the bones. Each vertex that makes up the skin is affected by one or more bones. When the vertex is affected by multiple bones, the different bones determine the weight of the impact on the vertex according to the geometric and physical relationship with the vertex. The correct position of the vertex in the world coordinate system is obtained by calculating the weighting of the effects of the different bones that affect the vertex. The key frame in the animation file usually holds the orientation of the bones, the position and other information, through the animation sequence in the adjacent two key frame interpolation can determine a certain moment of each bone new orientation and new position, and then according to the skin grid The affected bones index and weight data stored in the vertex calculate the new position of the vertex, enabling a single skin mesh deformation animation under the skeletal drive, or a skeletal skin animation. Bone skin animation effect than the joint animation and a single grid animation more realistic, more vivid. Moreover, with the improvement of 3D hardware performance, more and more related computing can be done through hardware, bone skin animation has become the use of all kinds of real-time animation applications, the most extensive animation technology. The method of surface model and skeleton driving deformation proposed in this paper is combined with skeleton skin animation technology to realize virtual human motion drawing.

\section{Acknowledgement}

This research was financially supported by the National Science Foundation.

\section{References}

[1] Yan.L, ZhaoJI.W, TianL.M。3D Virtual Human Body rendering mothed,Compute research and development, 2005, 42(5):888-896.

[2] N. Magnenat-Thalmann, R. Laperriere, and D. Thalmann. Joint-Dependent Local Deformations for Hand Animation and Object Grasping. In Proceedings of Graphics Interface,1988:26-33.

[3] S. Yoshimoto. Ballerinas Generated by a Personal Computer. The Journal of Visualization and Computer Animation,April, 1992, 3(1): 85-90.

[4] J. Shen. Human Body Modeling and Deformations. PHD. thesis, École Polytechnique Fédérale de Lausanne,1996. tion on http://www.weld.labs.gov.cn 